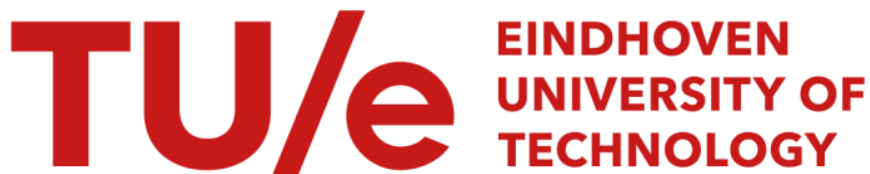

\section{Jet formation and jet velocity fluctuations in a flue organ pipe}

\section{Citation for published version (APA):}

Verge, M. P., Fabre, B., Mahu, W. E. A., Hirschberg, A., van Hassel, R. R., Wijnands, A. P. J., de Vries, J. J., \& Hogendoorn, C. J. (1994). Jet formation and jet velocity fluctuations in a flue organ pipe. Journal of the Acoustical Society of America, 95(2), 1119-1132. https://doi.org/10.1121/1.408460

DOI:

$10.1121 / 1.408460$

Document status and date:

Published: 01/01/1994

\section{Document Version:}

Publisher's PDF, also known as Version of Record (includes final page, issue and volume numbers)

\section{Please check the document version of this publication:}

- A submitted manuscript is the version of the article upon submission and before peer-review. There can be important differences between the submitted version and the official published version of record. People interested in the research are advised to contact the author for the final version of the publication, or visit the $\mathrm{DOI}$ to the publisher's website.

- The final author version and the galley proof are versions of the publication after peer review.

- The final published version features the final layout of the paper including the volume, issue and page numbers.

Link to publication

\section{General rights}

Copyright and moral rights for the publications made accessible in the public portal are retained by the authors and/or other copyright owners and it is a condition of accessing publications that users recognise and abide by the legal requirements associated with these rights.

- Users may download and print one copy of any publication from the public portal for the purpose of private study or research.

- You may not further distribute the material or use it for any profit-making activity or commercial gain

- You may freely distribute the URL identifying the publication in the public portal.

If the publication is distributed under the terms of Article 25fa of the Dutch Copyright Act, indicated by the "Taverne" license above, please follow below link for the End User Agreement:

www.tue.nl/taverne

Take down policy

If you believe that this document breaches copyright please contact us at:

openaccess@tue.nl

providing details and we will investigate your claim. 


\section{Jet formation and jet velocity fluctuations in a flue organ pipe}

M. P. Verge, B. Fabre, W. E. A. Mahu, A. Hirschberg, R. R. van Hassel, A. P. J. Wijnands, J. J. de Vries, and C. J. Hogendoorn

Citation: The Journal of the Acoustical Society of America 95, 1119 (1994); doi: 10.1121/1.408460

View online: https://doi.org/10.1121/1.408460

View Table of Contents: http://asa.scitation.org/toc/jas/95/2

Published by the Acoustical Society of America

\section{Articles you may be interested in}

Sound production in recorderlike instruments. I. Dimensionless amplitude of the internal acoustic field The Journal of the Acoustical Society of America 101, 2914 (1997); 10.1121/1.418521

Sounding Mechanism of the Flute and Organ Pipe

The Journal of the Acoustical Society of America 44, 983 (1968); 10.1121/1.1911240

Sound production in recorderlike instruments. II. A simulation model

The Journal of the Acoustical Society of America 101, 2925 (1997); 10.1121/1.419481

Jet drive mechanisms in edge tones and organ pipes

The Journal of the Acoustical Society of America 60, 725 (1976); 10.1121/1.381120

Time-domain simulation of flute-like instruments: Comparison of jet-drive and discrete-vortex models The Journal of the Acoustical Society of America 136, 389 (2014); 10.1121/1.4875716

Simplified models of flue instruments: Influence of mouth geometry on the sound source

The Journal of the Acoustical Society of America 113, 1724 (2003); 10.1121/1.1543929 


\title{
Jet formation and jet velocity fluctuations in a flue organ pipe
}

\author{
M. P. Verge \\ I.R.C.A.M., 1 Place Igor Stravinsky, 75004, Paris, France
}

B. Fabre

Laboratoire d'acoustique musicale, C.N.R.S., U.R.A. 868, Université de Paris VI, Tour 66, 4 Place Jussieu, 75252, Paris, France

W. E. A. Mahu, A. Hirschberg, R. R. van Hassel, A. P. J. Wijnands, J. J. de Vries, C. J. Hogendoorn

Technische Universiteit Eindhoven, W\&S 0.54, Postbus 513, 5600 Eindhoven, The Netherlands

(Received 22 March 1993; revised 27 September 1993; accepted 26 October 1993)

\begin{abstract}
Flow visualization of the initial transient in a small recorderlike flue organ pipe is presented and the various stages of the jet formation are related to measurements of the acoustic response of the pipe. An initial acoustic signal, due to the unsteady volume flow of the jet, appears before the forming jet reaches the labium. This signal can easily be modeled using a low-frequency approximation. The initial trajectory of the jet makes a curve towards the exterior of the pipe. Under certain conditions, the jet may even, at first, miss the labium. This effect is related to the steepness of the pressure rise in the foot of the pipe. The initial impact of the jet with the labium appears to be a crucial factor in the triggering of the transient. Moving the labium towards the exterior of the pipe, using a steep pressure rise or putting ears around the mouth increase the chance that the jet will hit the labium. This initial impact is followed by an impulsive vortex shedding at the labium and subsequently a high-frequency varicoselike oscillation is observed on the jet. This oscillation is also observed without labium. After about three periods of the fundamental mode of the pipe, turbulence appears therefore destroying these coherent structures. Whereas the time dependency of the jet velocity dominates the first stage of the starting transient, the jet velocity fluctuations during steady-state result in a non-negligible damping. This loss mechanism is, for the fundamental mode of our experimental organ pipe, of the same order of magnitude as the radiation or visco-thermal damping.
\end{abstract}

PACS numbers: $43.75 . \mathrm{Np}$, 43.28. Ra

\section{INTRODUCTION}

The aim of the present research is to develop physical models of flutelike musical instruments suitable for timedomain simulations. The work presented in this paper is based on experimental measurements which are described in the following sections. We will mainly focus on the initial transient. Indeed, this regime appears to be crucial from the point of view of perception and therefore represents an important element of a good simulation. We concentrate on the first stage of the transient at the moment at which the jet formation results, by volume injection into the system, in a variable volume flux into the pipe and, hence, the formation of an initial acoustic wave at its entrance. In order to describe our observations of the response of the pipe during the first phase of the initial transient, we propose a simple physical model which is used for time-domain simulations. We then present calculations of the jet formation time obtained with different methods and a simple model to describe the trajectory of the forming jet. In the literature, a constant jet velocity is assumed during steady state. However, we show that the velocity variations during this regime are significant enough to induce a nonnegligible damping of the oscillation in the pipe. It is interesting to note that in spite of the simple nature of many of the effects which are described in this paper, they have not been considered before in the literature.
Flue organ pipes are usually modeled as a feedback loop composed of a linear resonator coupled to an excitator, the jet-edge system. ${ }^{1-4}$ The jet itself is assumed to be a linear amplifier which responds to the acoustic flow at the flue exit. The nonlinearity of the system, which is necessary to reach a finite oscillation amplitude upon steady-state operation, is commonly assumed to be a saturation of the jet drive when the lateral displacement of the jet at the labium becomes larger than the jet width. While this theory is considered to be fairly accurate, it nevertheless fails to predict important parameters: The predicted blowing pressure threshold for steady-state oscillation is approximately a factor three too high ${ }^{5}$ and the amplitude of the fundamental mode of the acoustic oscillation is overestimated by one order of magnitude. ${ }^{6}$

The study of Fabre ${ }^{6}$ shows that acoustically induced vortex shedding at the labium, which corresponds to the amplitude dependency of the mouth impedance reported by Coltman, ${ }^{7}$ constitutes a very crucial nonlinear damping much more important than the linear visco-thermal or radiative losses considered by Fletcher. ${ }^{2,5}$ As suggested by Fabre, this impulsive vortex shedding at the labium which occurs at high blowing pressure also largely determines the generation of higher harmonics and hence, the musical quality of the sound. In this sense, the use of a vortex sound theory focusing on the flow around the labium, as 


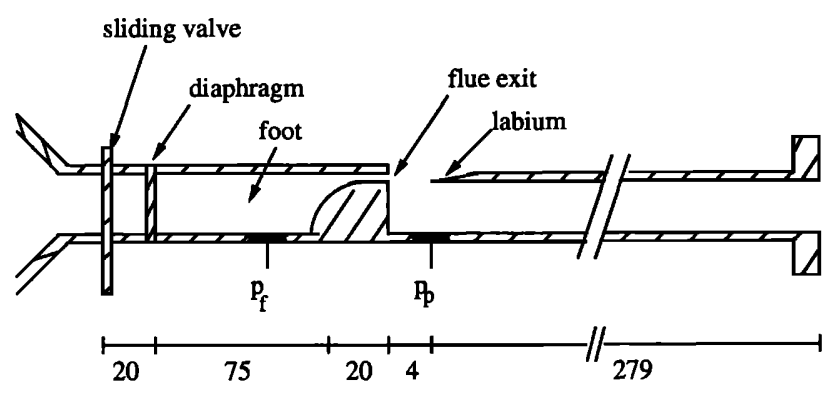

FIG. 1. Experimental organ pipe (dimensions in $\mathrm{mm}$ ).

proposed by Howe, ${ }^{8}$ appears to be a powerful approach. However, the vortex shedding yields a damping of the fundamental rather than an amplification as suggested by Howe. The experimental measurements presented in this paper will show that vortex shedding at the labium also drastically affects the initial transient. We will however leave the discussion of this aspect of the problem for a subsequent paper. At the present time, we will only focus on the formation of the jet. We should finally stress that we are only able to propose qualitative information for the observations that we present.

\section{EXPERIMENTAL PROCEDURES}

In our experimental study, at the Eindhoven University of Technology, we have used a small organ pipe with a geometry typical of dutch street pipe organs and renaissance recorders. The use of an organ pipe instead of a flute eliminates the human factor, which is convenient from an experimental point of view. For reasons of simplicity, we have also chosen a geometry with sharp edges and a straight flue channel. This does not correspond to a musically optimal configuration. Nevertheless, the features described in this paper are very general and are expected to appear in very different types of musical instruments.

The geometry of our flue organ pipe is shown in Fig. 1. The pipe is $283.0 \pm 0.1 \mathrm{~mm}$ long and is open at its passive extremity. The upper wall at the mouth of the pipe has an edge with an angle of $15^{\circ}$ which forms the labium. The pipe width $H$ is $20.00 \pm 0.05 \mathrm{~mm}$. The walls are made out of brass and are $5 \mathrm{~mm}$ thick. The foot of the pipe has a length of $75 \mathrm{~mm}$ and a volume $V$ of $34 \mathrm{~cm}^{3}$. It is connected to the mouth of the pipe by a $20-\mathrm{mm}$-long channel of variable height $h$ and whose width $H$ is the same as that of the pipe. At the flue exit, the height $h_{e}$ of the channel is equal to $1.01 \pm 0.02 \mathrm{~mm}$. The lower edge of the flue exit is located $19.92 \pm 0.01 \mathrm{~mm}$ above the lower wall of the pipe. The distance $W$ between the edge of the labium and the flue exit is $4.05 \pm 0.05 \mathrm{~mm}$, the mouth surface $S_{m}$ is therefore equal to $81 \mathrm{~mm}^{2}$. The upper wall of the pipe (or lower part of the labium) was placed at a distance $y_{0}$ equal to $0.30 \pm 0.02$ $\mathrm{mm}$ above the lower wall of the flue channel. Hence the pipe cross-section area $S_{p}$ is $404.40 \mathrm{~mm}^{2}$. Typical radii of curvature of the edges in the organ pipe are $0.03 \mathrm{~mm}$ at the labium and $0.02 \mathrm{~mm}$ for the edges of the flue channel exit.

The foot is delimited upstream by an 8-mm-diam circular diaphragm covered with a fine gauze with a mesh size of about $1 \mathrm{~mm}$. The aim of this diaphragm and gauze is to damp out the oscillations of the pressure $p_{f}$ in the foot. The air entering the pipe is delivered to the foot by a $10-\mathrm{cm}-$ long pipe with a diameter of $2 \mathrm{~cm}$ fixed on a $8-\ell$ settling chamber. This chamber is connected through a 10 -m-long pipe with an inner diameter of $19 \mathrm{~cm}$ to a large pressure supply room of $3600 \mathrm{~m}^{3}$. The room in which the setup is placed has a volume of $47 \mathrm{~m}^{3}$. The pressure difference between this room and the pressure supply is maintained by the ventilation system of the building (stability $0.5 \mathrm{~Pa}$ ). The flow is triggered by opening a valve with a diameter of $2 \mathrm{~cm}$ just upstream of the foot of the pipe. This valve consists of a small wooden plate which is driven like an arrow by a crossbow. It can be pulled mechanically when the crossbow is used; the typical rise time $\tau$ of the pressure $p_{f}$ is then of about $0.5 \mathrm{~ms}$ and the experiment can be reproduced accurately. For a smoother pressure rise $(\tau \approx 10$ ms) the valve can be pulled manually. In this case the reproducibility of the experiments is poor and hence the data of different experiments are not accurately correlated.

The pressure $p_{f}$ in the foot of the pipe and the pressure $p_{p}$ at its entrance (just below the mouth, $277.80 \mathrm{~mm}$ from the end of the pipe) are measured by using accelerationcompensated piezoelectrical gauges (type PCB $116 \mathrm{~A}$ ). The gauges are mounted flush in the walls and their position is shown in Fig. 1. The charge delivered by the gauges is amplified by means of charge amplifiers (Kistler type 5007, with a bandwidth extending between $0.1 \mathrm{~Hz}$ and 22 $\mathrm{kHz}$ ) and transmitted to a digital memory ( 8 bits, 2048 samples) for further analysis with a PC. The pressure gauges were calibrated with an accuracy of $1 \%$. Flow visualization through glass windows placed around the mouth are done using a Schlieren technique. ${ }^{9}$ A refractive index difference between the gas flowing out of the flue exit and the air in the pipe is obtained by filling the foot of the pipe with $\mathrm{CO}_{2}$ prior to each experiment. The light source is a Nanolite spark discharge with a duration of 80 ns. The light spark is triggered by the output of a delay line driven by the pressure signal $p_{f}$. For each experiment, a single picture of the flow is taken at a given instant determined with an accuracy of $10 \mu \mathrm{s}$. This procedure is then repeated for different delays in order to obtain the series of pictures shown in Figs. 4-6. As noted above, when the valve is driven by the crossbow the transient can be reproduced. For manual openings of the valve, the sequence of pictures shown do not correspond to identical pressure signals and can only be compared qualitatively. However, the difference between the two types of transient is so dramatic that even such a comparison is interesting. Please note that as pure $\mathrm{CO}_{2}$ is used, the gas density in the flue channel is significantly changed in comparison to the density of air and this has to be taken into account in our theoretical analysis (we use the density of $\mathrm{CO}_{2}$ ). However, the organ pipe itself is free from $\mathrm{CO}_{2}$ so that its acoustical properties are not modified. Furthermore, experiments and theoretical analysis by Fabre $^{6}$ show that $\mathrm{CO}_{2}$ injection does not significantly modify the sound production in the pipe. 

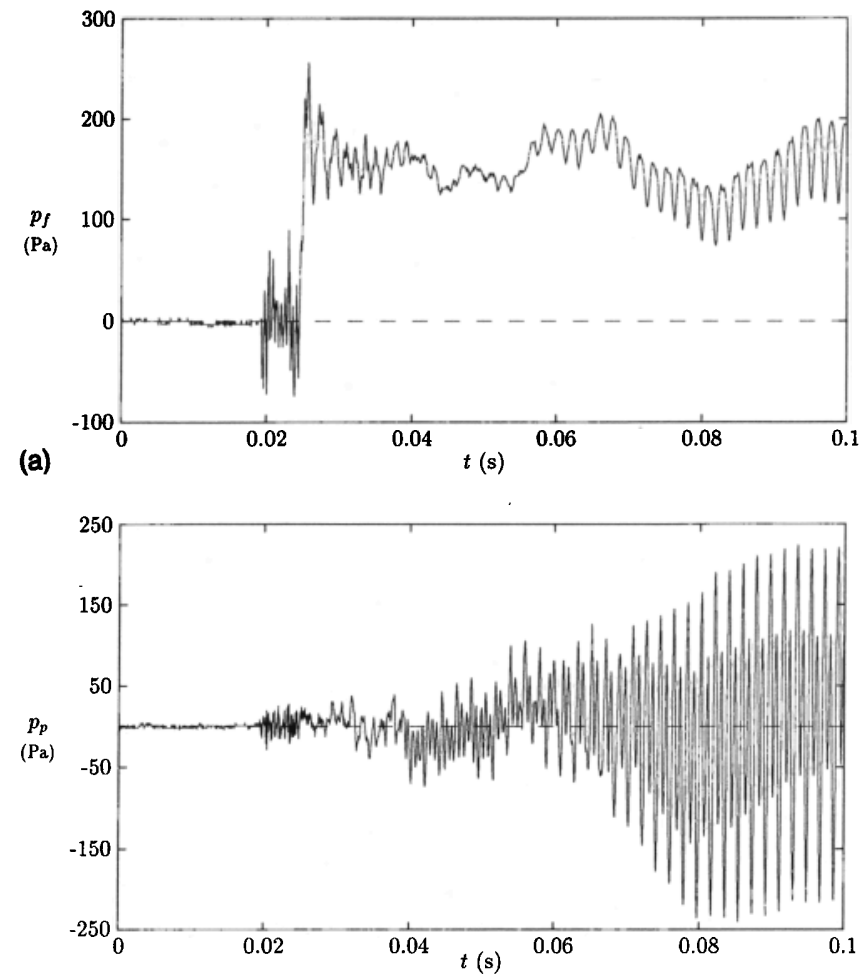

(b)

FIG. 2. Measured pressure signal for a fast valve opening: (a) in the foot of the pipe; (b) at the entrance of the pipe.

\section{PRESSURE SIGNALS}

The initial rise of the pressure $p_{f}$ in the foot of the pipe is controlled by the speed of the opening of the valve. Pressure signals measured in the foot for a fast and a slow opening of the valve are shown in Figs. 2(a) and 3(a). A steep pressure rise with a characteristic rise time of $0.5 \mathrm{~ms}$ is obtained by opening the valve with the crossbow. As seen in Fig. 2(a), the pressure history shows an overshoot of about $25 \%$ of the steady-state mean value followed by oscillations at about $1 \mathrm{kHz}$ which are damped out within approximately $15 \mathrm{~ms}$. The pressure rise is preceded by a high-frequency noise generated by the friction of the wooden valve driven by the crossbow. This noise does not appear to have a strong influence on the jet velocity response but may limit the generality of the observations we will make. A much smoother pressure rise is obtained with a manual opening of the valve. The typical rise time is then of the order of $10 \mathrm{~ms}$. This time can be considered as being relatively long compared to the round trip time of a wave traveling in the resonator (about $2 \mathrm{~ms}$ for our experimental flue pipe). Hence we have, with the mechanical and manual opening of the valve, two sets of experiments corresponding to extreme situations. In typical playing conditions, the rise time is expected to lie within these two limits. $^{10}$

Measurements of the pressure signal $p_{p}$ at the pipe entrance corresponding to the driving pressure histories of Figs. 2(a) and 3(a) are shown in Figs. 2(b) and 3(b), respectively. A dramatic dependence of the pipe pressure response on the foot pressure rise time is observed. When a steep pressure rise is used, the formation of a small pres-
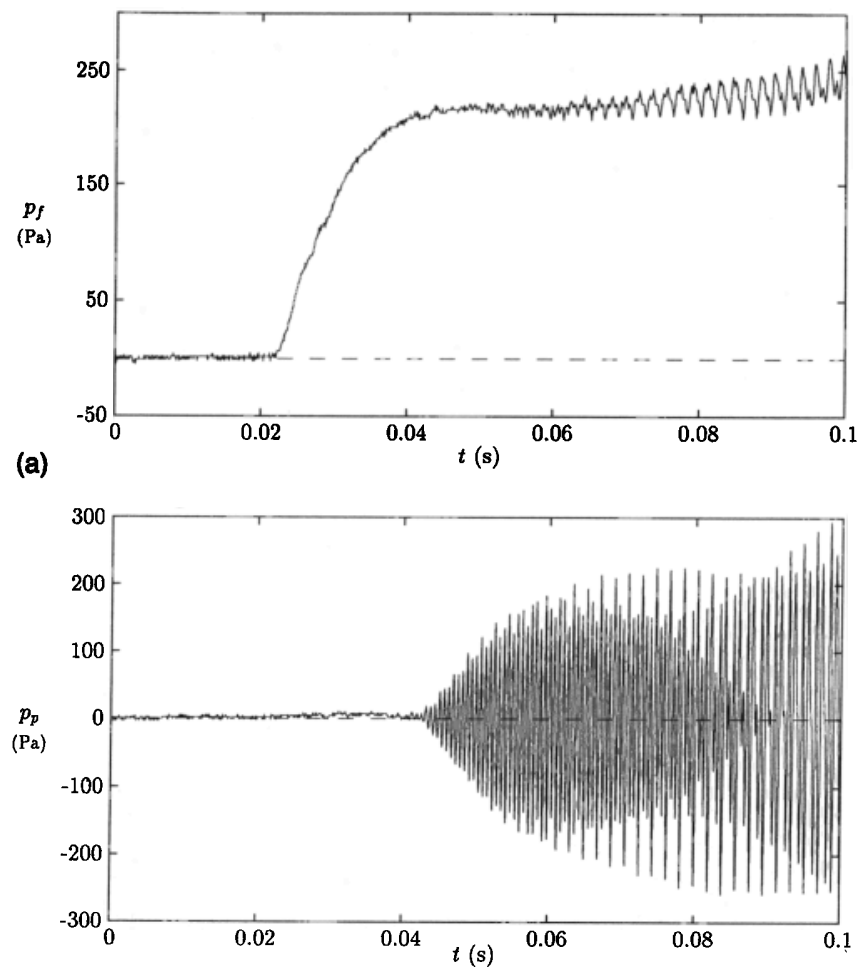

(b)

FIG. 3. Measured pressure signal for a slow valve opening: (a) in the foot of the pipe; (b) at the entrance of the pipe.

sure pulse with a duration of about $2 \mathrm{~ms}$ is noticed just after the opening of the valve. As will be seen later, this first pressure pulse is due to the initial acceleration of the jet which results in an unsteady volume flow into the pipe. This pulse is difficult to see at first glance because it is preceded by noise due to the friction of the valve. However, as seen in Figs. 10(b) and 11(b), this pulse reproduces well and its magnitude appears to correspond to the theoretical prediction which we will discuss further. Some $4 \mathrm{~ms}$ after the start of the opening of the valve, pressure peaks are observed. This is followed, after some complex initial pressure response, by a periodic sequence of peaks at regular time intervals (about $2 \mathrm{~ms}$ ) that correspond to the fundamental mode of the pipe. For steep driving pressure rises, the initial oscillation in the pipe is often dominated by a frequency corresponding to the second or third mode of the pipe. After an exponential growth of the oscillation, saturation occurs along with a shift towards a lower acoustic mode of the pipe. ${ }^{6,10-12}$ Below $190 \mathrm{~Pa}$, our experimental flue pipe sounds at its fundamental mode under steadystate conditions, while above $270 \mathrm{~Pa}$ the second mode prevails. It is interesting to note that the amplitude of the steady-state pressure signal $p_{p}$ is comparable to the average pressure $p_{f}$ in the foot. As will be seen in Sec. IV E this results in jet velocity fluctuations which are not negligible. A consequence of these jet velocity fluctuations is that they induce pressure variations in the foot of the pipe. This is clearly seen on the pressure signals of Figs. 2(a) and 3(a), where an oscillation of the same frequency as that of the acoustic signal in the resonator is observed.

The pressure history for a manual opening of the valve 
(a)

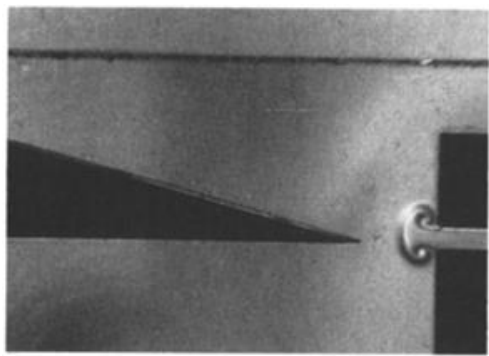

(b)

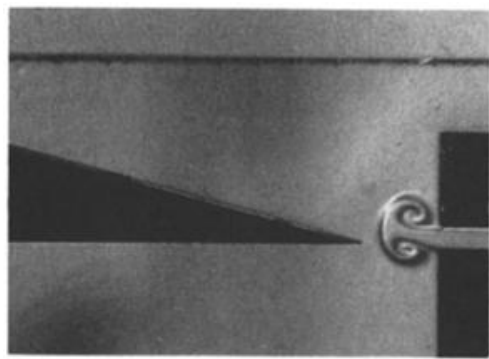

(c)

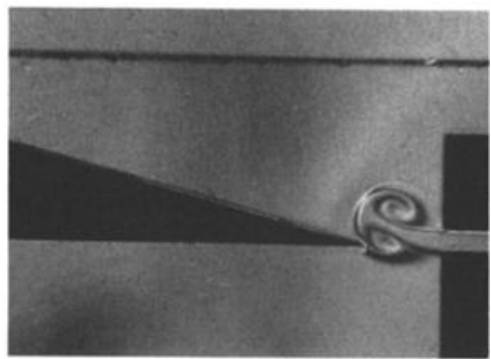

(d)

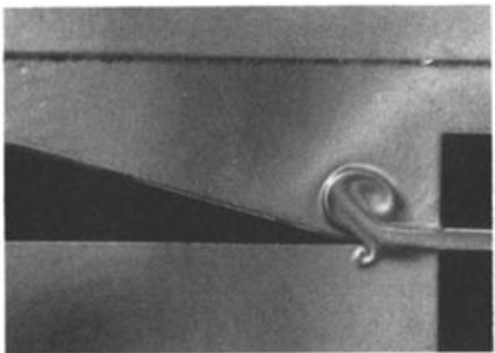

(e)
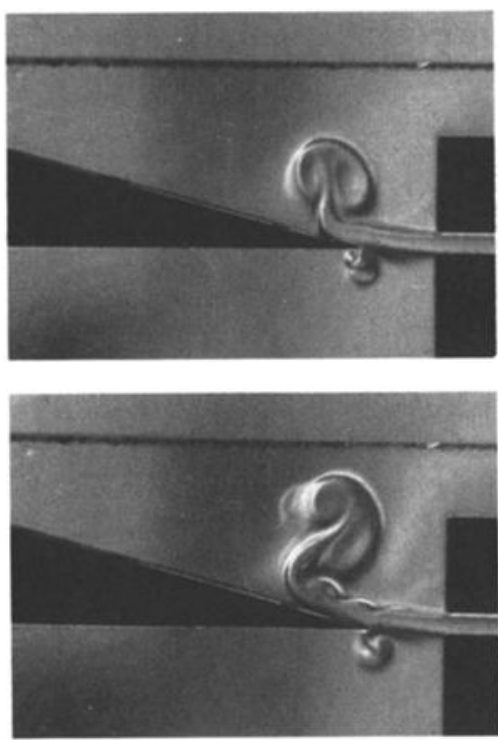

(f) (g)

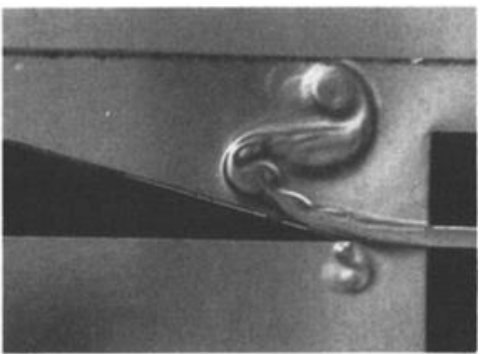

(h)
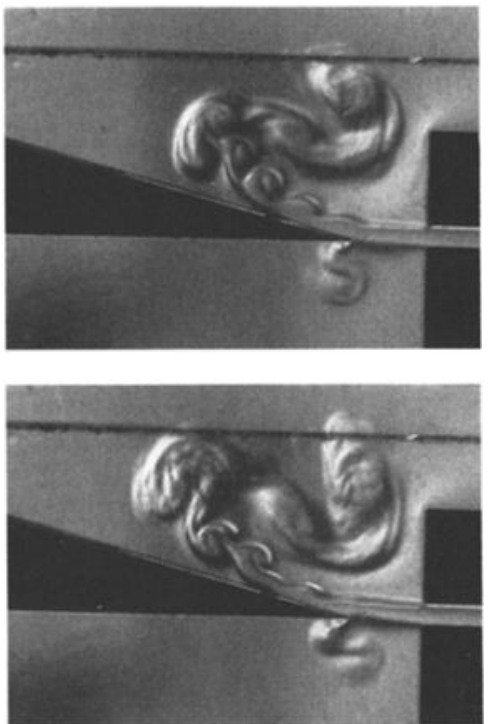

(i)

FIG. 4. Flow visualization at the mouth of the pipe for the steep pressure rise in the foot of the pipe. The different pictures were taken 1.69 , $1.98,2.49$, $3.03,3.50,4.13,4.44,5.12$, and $5.56 \mathrm{~ms}$, respectively, after the opening of the valve.

is quite different from the one we have just discussed for the mechanical opening. With a smooth pressure rise, the initial pressure pulse induced by the variable volume flux during the formation of the jet is faint yet clearly observable. It is followed by about $20 \mathrm{~ms}$ of relative silence. The acoustic oscillation in the pipe then starts and is dominated by the third mode of the pipe. In contrast with the behavior induced by the fast valve opening, the growth of the acoustic oscillation is smooth. The saturation amplitude is reached after about 30 oscillations and it then slowly shifts towards the fundamental mode frequency.

Similar measurements carried out on an alto recorder showed that the response of the instrument depends on the same way on the steepness of the driving pressure rise measured in the mouth of the musician. ${ }^{13}$ It is very interesting to see that observations made on an experimental organ pipe are also found on a musical instrument and, therefore, seem to have a certain generality.

\section{FLOW VISUALIZATION}

The flow visualization corresponding to the driving pressure signals of Figs. 2(a) and 3(a) are shown in Figs. 4 and 5, respectively. For the fast opening, we show the flow at intervals of approximately $0.5 \mathrm{~ms}$ during the first 6 ms. For the slow valve opening, we show the first $10 \mathrm{~ms}$ with time intervals of $1.5 \mathrm{~ms}$. On the first picture of Figs. 4 and 5, it is seen that the flow at the flue exit separates from the walls at the flue exit forming a pair of vortices. The flow separation at the sharp edges is due to friction. Initially, the two vortices grow independently and their paths make an angle of about $45^{\circ}$ with the flue axis. After the vortices have traveled a distance comparable with the flue exit width $h_{e}$, the horizontal velocity which they induce on each other becomes dominant. The two vortices travel towards the labium as they grow further. At this point it is clearly observed that the vortex pair and the jet which is formed behind it do not follow a straight path along the flue channel axis. Indeed, the vortex pair trajectory makes a curve towards the exterior of the mouth. This effect is much more pronounced for the slow valve opening than for the fast one. This flow deflection is expected to be a consequence of the asymmetry between the flow distribution at the flue exit; a more quantitative discussion of this effect is given in Sec. V B.

Due to the strong deflection when a slow opening of the valve is used, the vortex pair at the front of the jet passes at some distance from the labium. A strong interaction of the jet with the labium is, therefore, delayed. The flow visualization shows that in these conditions the jet touches the labium some $4 \mathrm{~ms}$ later. When the fast opening is used, the jet hits the labium at once about $2 \mathrm{~ms}$ after the opening of the valve. As was discussed in the Introduction, the impact of the jet with the labium is an event that generates an acoustic wave at the entrance of the pipe since it 
(a)
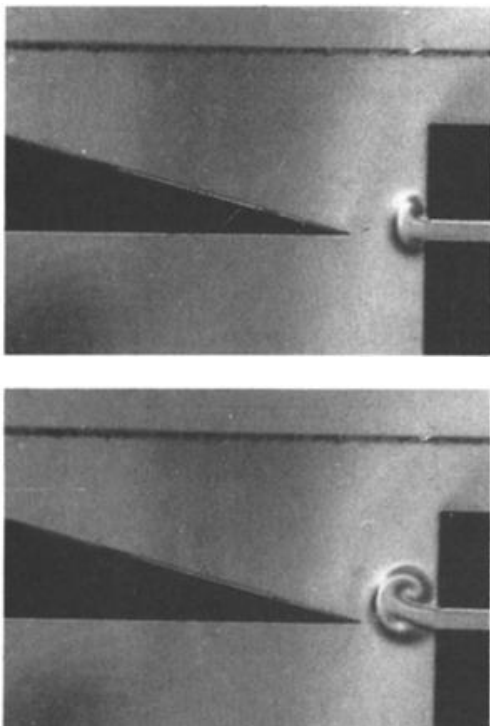

(b)

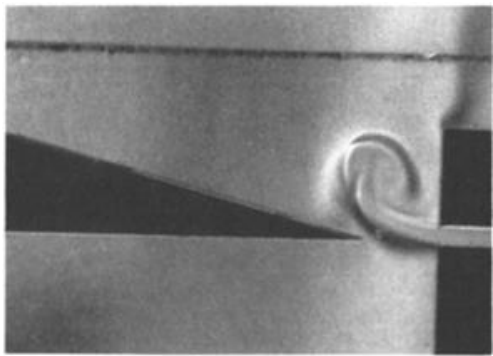

(c)

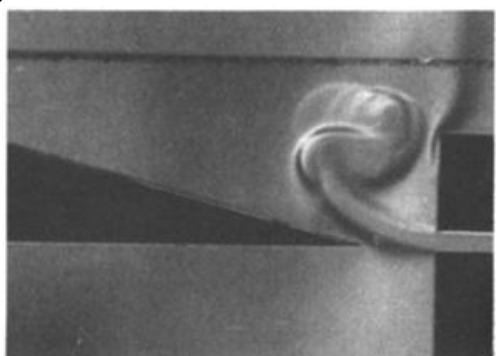

(d)

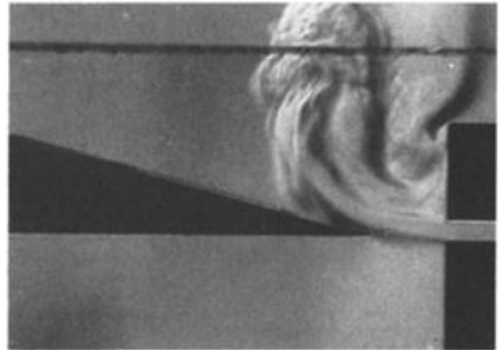

(e)

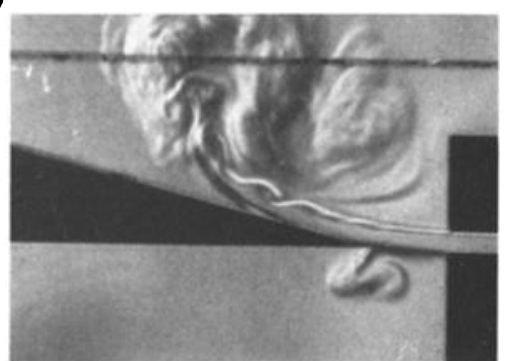

(f)

FIG. 5. Flow visualization at the mouth of the pipe for the slow pressure rise in the foot of the pipe. The different pictures were taken $2.5,4.0,5.5,7.0$, 8.5 , and $10.0 \mathrm{~ms}$, respectively, after the opening of the valve.

results in the shedding of a vortex. ${ }^{14}$ We indeed observe strong fluctuations in the pipe response after the jet has reached the labium. The contact of the jet with the labium therefore seems to be an important element in the triggering of the transient. It is however difficult to relate systematically the various flow observations to a particular pressure response. Indeed, the flow complexity is further increased by the appearance of a varicose type oscillation of the jet with a frequency close to the most unstable frequency of a planar jet as observed by Sato $^{15}$ $\left(\mathrm{Sr}=f h_{j} / v_{j}=0.23\right.$, where $\mathrm{Sr}$ is the Strouhal number, $h_{j}$ the jet height, $f$ the frequency, and $v_{j}$ the jet speed). This oscillation around 3 to $4 \mathrm{kHz}$ may correspond to an edgetone feedback loop. ${ }^{16}$ While, as is seen in Fig. 6, similar instabilities are observed also in experiments in the absence of a labium, there is a clear correlation between the onset of these instabilities and the contact of the jet with the labium. It is also unlikely that these oscillations are induced by the oscillation of the pressure $p_{f}$ in the foot of the pipe since we do not observe a significant signal around $3 \mathrm{kHz}$ in the foot. Furthermore, such high frequencies would be integrated by the large inertia of the air in the long flue channel used in our experiments.

It is interesting to note that we do not observe a strong pipe response at the frequency corresponding to the first transversal pipe mode. This contrasts with earlier experiments ${ }^{17}$ where we did observe such a high-frequency response during the initial transient. Nolle ${ }^{10}$ and Castellengo $^{18}$ have also observed high-frequency bursts during the first milliseconds of organ pipe and recorder responses which may be related to an edge-tone phenomenon. In particular, Castellengo obtained such bursts in a recorder both with and without a pipe. ${ }^{6}$

After 4 or $5 \mathrm{~ms}$ turbulence gradually appears thus washing out the coherent two-dimensional structures on that portion of the jet moving away from the labium. After the contact between the jet and the labium has been established, transversal oscillations of the jet are observed. Because we consider here an asymmetric labium position, the jet only interacts with the edge of the labium when it is in its most inward position. Vortices are then shed to which correspond sharp acoustic pulses in the pipe response. This represents a highly nonlinear interaction between the jet and the labium even for an oscillation amplitude small compared to the jet width. This nonlinearity certainly strongly encourages the oscillation of higher-order modes of the pipe during the initial phase of the transient.

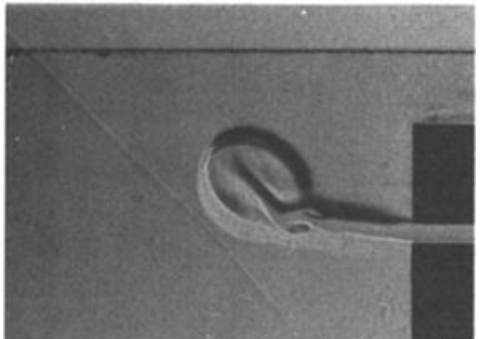

(a)

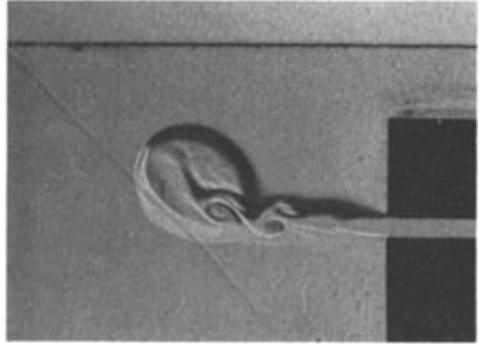

(b)

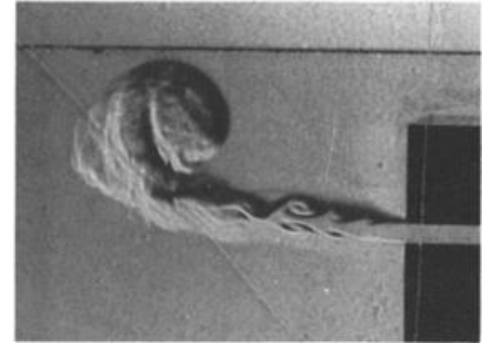

(c)

FIG. 6. Flow visualization of the forming jet without labium (only the upper wall of the pipe is removed). 


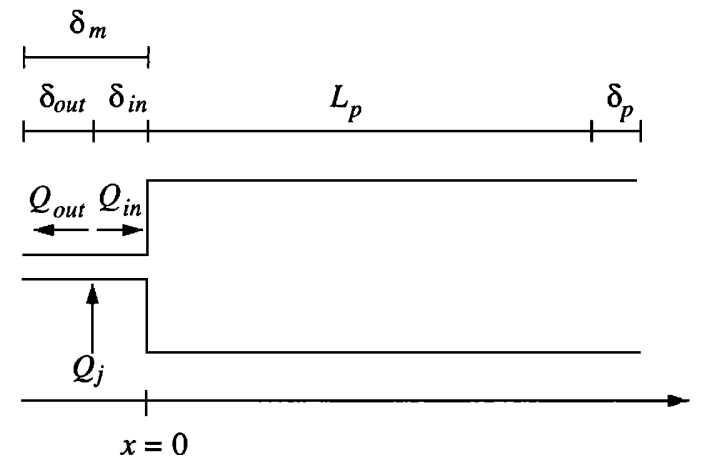

FIG. 7. Simple acoustic model.

\section{PIPE RESPONSE TO JET VOLUME FLUX}

\section{A. Simple acoustic model}

Our analysis of the effects of the initial volume injection into the mouth of a flue organ pipe is based on the simple one-dimensional acoustic model presented in Fig. 7. The tube on the left represents a region around the flue exit small enough, compared to the wavelength of the acoustic movements considered, to neglect locally the effects of compressibility. The cross section $S_{m}$ of this tube is equal to that of the mouth and its length $\delta_{m}$ represents the end correction that can be associated with the mouth. The larger tube on the right, having the same cross section $S_{p}$ and length $L_{p}$ as that of the pipe, represents the resonator where propagation phenomena take place. The flue exit, where flow injection occurs, is located at coordinate $x=-\delta_{\text {in }}$ with $x$ an axis parallel to the tubes. Flow and pressure continuity is assumed at the interface between the two tubes.

The end correction $\delta_{m}$ can be determined theoretically by assuming that it consists of three terms: ${ }^{19}$

$$
\delta_{m} \approx \delta_{c}+\delta_{e}+\delta_{r}
$$

The first term $\delta_{c}$ is the contribution due to the mouth constriction. If we consider this constriction as a diaphragm with an opening of rectangular surface $W H$ placed asymmetrically in a pipe of cross section $H^{2}$, we can calculate the correction $\delta_{c}$ with the expression proposed by Morse and Ingard: ${ }^{20}$

$$
\delta_{c}=\frac{4}{\pi} W \ln \left(\frac{1}{2} \tan \frac{\pi W}{4 H}+\frac{1}{2} \cot \frac{\pi W}{4 H}\right) .
$$

The second term $\delta_{e}$ represents the effects of the glass windows placed around the mouth and acting as ears. In our experimental pipe, they exceed the flue exit axis by a length $l_{e}=9.5 \mathrm{~mm}$ and delimit a surface approximately equal to that of the pipe. The last term $\delta_{r}$ corresponds to the inertia of the flow at the end of the glass windows.

In order to express the end corrections $\delta_{e}$ and $\delta_{r}$ in length of pipe having a cross section equal to that of the mouth, we use the following expression which converts a length of pipe $\Delta l$ having a cross section $S_{p}$ into an end correction $\delta$ of cross section $S_{m}$ :

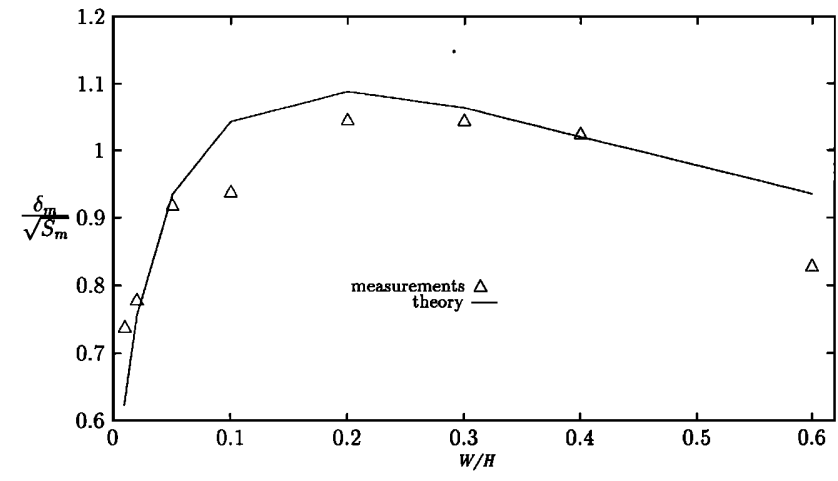

FIG. 8. Measured and calculated total end correction $\delta_{m}$ of the mouth.

$$
\delta=\frac{S_{m}}{S_{p}} \frac{\tan k_{0} \Delta l}{k_{0}},
$$

where $k_{0}=\omega / c_{0}$ is the wave number, with $c_{0}$ the speed of sound in air. Equation (3) can be deduced from a lowfrequency approximation of the resonance condition of the acoustic model of Fig. 7:

$$
\tan k_{0}\left(L_{p}+\delta_{p}\right)=-\frac{S_{p}}{S_{m}} k_{0} \delta_{m},
$$

where $\delta_{p}$ is an end correction at the passive extremity of the pipe. Measurements by means of a two-microphone method show that our experimental pipe shows a flanged pipe behavior with $\delta_{p}$ equal to ${ }^{21}$

$$
\delta_{p}=0.82 \sqrt{\frac{H^{2}}{\pi}} .
$$

Finally, using Eq. (3) and assuming a flanged pipe behavior at the mouth, we obtain for the end corrections $\delta_{e}$ and $\delta_{r}$ :

$$
\begin{aligned}
\delta_{e} & =\frac{S_{m}}{S_{p}} \frac{\tan k_{0} l_{e}}{k_{0}}, \\
\delta_{r} & =\frac{S_{m}}{S_{p}} \frac{\tan k_{0}\left(0.82 \sqrt{H^{2} / \pi}\right)}{k_{0}} .
\end{aligned}
$$

The values of the total end correction $\delta_{m}$ at the mouth calculated from Eqs. (1), (2), (6), and (7) are shown in Fig. 8 for the fundamental passive resonance frequency of our pipe and different ratio $W / H$. They are compared with the corresponding experimental values of the end correction of the mouth deduced from the measurement of the passive resonance frequencies $f_{\text {res }}$ of our experimental pipe, Eq. (3) and the following:

$$
\Delta l=\left(\frac{c_{0}}{2 f_{\text {res }}}-L_{p}-\delta_{p}\right) .
$$

The calculated values are fairly accurate and predict the dependency of the end correction with the mouth geometry.

The end correction associated with the mouth of the acoustic model of Fig. 7 was calculated from Eq. (1) for the fundamental passive resonance frequency of our pipe. In Table I we compare the resonance frequencies of our 
TABLE I. Measured and calculated passive resonance frequencies of our experimental organ pipe.

\begin{tabular}{ccc}
\hline \hline $\begin{array}{c}\text { Measured resonance } \\
\text { frequencies } \\
(\mathrm{Hz})\end{array}$ & $\begin{array}{c}\text { Resonance frequencies } \\
\text { calculated with Eq. (4) } \\
(\mathrm{Hz})\end{array}$ & $\begin{array}{c}\text { Resonance frequencies } \\
\text { with losses } \\
(\mathbf{H z})\end{array}$ \\
\hline 514 & 514 & 514 \\
1057 & 1044 & 1044 \\
1597 & 1591 & 1592 \\
2157 & 2152 & 2154 \\
2741 & 2720 & 2725 \\
3292 & 3294 & 3303 \\
3913 & 3871 & 3886 \\
4460 & 4450 & 4472 \\
5063 & 5031 & 5062 \\
5657 & 5613 & 5653 \\
6240 & 6195 & 6247 \\
\hline
\end{tabular}

model calculated with Eq. (4) and the measured passive resonance frequencies of our experimental pipe for a ratio $W / H=0.2$. We also include in Table I resonance frequencies obtained by taking into account radiation and viscothermic losses. These frequencies correspond to the minima of the impedance curve at the end of the tube and were calculated by using a complex propagation constant ${ }^{22}$ and the real part of the radiation impedances. These results show that the end correction $\delta_{m}$ should be adjusted with frequency in order to fit the experimental data exactly. However, in this paper, this effect will be neglected and the end correction $\delta_{m}$ will be considered to be constant. A very significant advantage of the theoretical model is that in analogy to $\mathrm{Howe}^{8}$ we have a potential flow model which not only can be used to calculate global acoustical properties of the mouth (like the end correction at the mouth) but also yields information about the nonuniformity of the flow. We will show that this model can also be used when considering the initial jet deflection (Sec. V B).

\section{B. Jet velocity}

The key element in the acoustic model presented in the previous section is that it takes into account the velocity variations of the flow at the flue exit during the formation of the jet. This enables one to simulate the pressure pulse seen on the initial pressure response $p_{p}$ of the resonator and the flow distribution at the flue exit. These features constitute important parameters to consider in a description of the transient. Indeed the initial pulse is the first acoustic signal to which the resonator responds and the flow distribution at the flue exit accounts for the dependence of the jet trajectory on the steepness of the driving pressure rise.

The injection of fluid into the mouth is driven by the pressure difference between the foot and the mouth of the pipe. Since the channel length is small compared to the distance traveled by sound waves during typical driving pressure rise times, the air in the channel can be considered to be incompressible. If friction is neglected in the flue channel, the flow velocity at the flue exit can be estimated by applying the Bernoulli equation ${ }^{23}$ between a point at the entrance of the channel and a point at the flue exit. It is

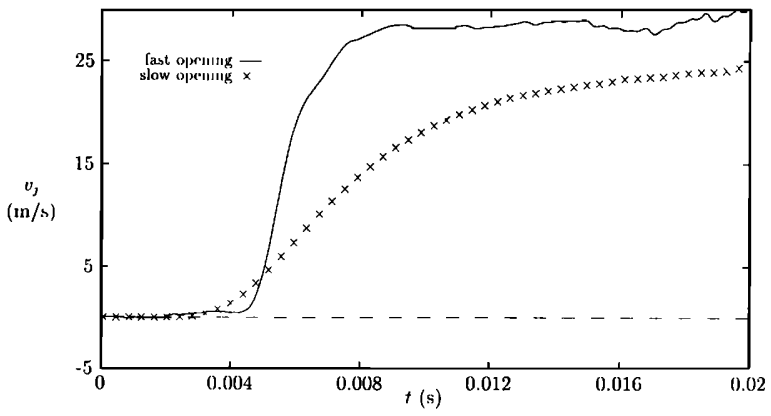

FIG. 9. Jet velocity at the flue exit for a fast valve opening and a slow valve opening.

further assumed that due to the flow separation (jet formation), the pressure at the flue exit is equal to the acoustic pressure $p_{m}$ in the mouth. Neglecting the air velocity in the foot of the pipe, the following relationship is obtained:

$$
\rho_{0} \frac{\partial\left(\varphi_{m}-\varphi_{f}\right)}{\partial t}+\frac{1}{2} \rho_{0} v_{j}^{2}=p_{f}-p_{m},
$$

where $t$ is time, $\rho_{0}$ is the air density, $\varphi_{m}, \varphi_{f}, p_{m}$, and $p_{f}$ the velocity potential and the pressure in the mouth and the foot of the pipe, respectively, and $v_{j}$ the jet velocity at the flue exit. In order to calculate the velocity potential difference, the velocity $v(y)$ of the flow along the flue channel must be known. If flow incompressibility and a uniform velocity across the channel width are assumed, we obtain from the mass conservation law:

$$
v(y)=\frac{v_{j} h_{e}}{h(y)},
$$

where $y$ is an axis parallel to the flue channel, $h(y)$ is the height of the flue channel decreasing from the foot to the flue exit, and $h_{e}$ is its height at the flue exit. Using Eq. (10), the difference of potential between the entrance of the channel and the flue exit can now be written

$$
\varphi_{m}-\varphi_{f}=\int_{f}^{m} v(y) d y=v_{j} h_{e} \int_{f}^{m} \frac{d x}{h(y)}=l_{c} v_{j},
$$

where $l_{c}$ is the effective channel length. For the geometry of our experimental pipe, a numerical evaluation of the integral of Eq. (11) yields a length $l_{c}$ of $2.7 \mathrm{~cm}$. From Eqs. (9) and (11), the following relationship is obtained:

$$
\rho_{0} l_{c} \frac{d v_{j}(t)}{d t}+\frac{1}{2} \rho_{0} v_{j}^{2}=p_{f}-p_{m} .
$$

Initially, the inertial term on the left-hand side of Eq. (12) dominates. Gradually, as the pressure in the foot stabilizes, the jet acquires kinetic energy density which, during steady state, is just equal to the pressure difference between the foot and the mouth of the pipe.

Typical velocity rises for a mechanical and a manual opening of the valve are shown in Fig. 9. They were obtained numerically from Eq. (12) and the experimental data presented in Figs. 10 and 11. In both cases, the velocity rises gradually; the integration effect of the inertia of the air in the flue channel constitutes a smoothing process of 



(b)

FIG. 10. Measured and simulated pressure signal for a fast valve opening: (a) measured pressure signal in the foot of the pipe; (b) measured pressure signal at the entrance of the pipe and corresponding simulated pressure signal.

the driving pressure fluctuations. In the rest of this paper, the jet volume flux $Q_{j}$ will be estimated to be equal to $h_{e} H v_{j}$ which neglects the influence of friction on the walls of the flue channel. The order of magnitude of the error in $Q_{j}$ due to friction is $2 \sqrt{v t / 3} / h_{e}$, where $v$ is the kinematic viscosity of air and is equal to $1.5 \times 10^{-5} \mathrm{~m}^{2} / \mathrm{s}$. This error is about $20 \%$ after $2 \mathrm{~ms}$.

\section{Initial plpe response}

The first acoustic signal appearing on the pressure signals $p_{p}$ measured at the entrance of the pipe and shown in Figs. 2(b) and 3(b) is a small positive pressure pulse. This initial acoustic signal is more obvious on the pressure signals of Figs. 10(b) and 11(b), where a larger time scale and a higher driving pressures were used [see Figs. 10(a) and 11(a)]. The formation of this pulse corresponds with the initial volume injection at the flue exit and is a consequence of the unsteady flow velocity at the flue exit. In order to estimate the amplitude of this initial signal, the flow distribution at the flue exit must first be determined.

At the origin, the mass conservation law gives

$$
Q_{j}=Q_{\text {out }}+Q_{\text {in }} \text {, }
$$

where $Q_{j}$ is the flow coming out of the flue exit, $Q_{\text {out }}$ the flow going towards the exterior of the pipe through the mouth and $Q_{\text {in }}$ the flow going inwards. The jet flow $Q_{j}$ is calculated with the Bernoulli equation. If nonlinear terms are neglected initially, which is valid for times such that inertia dominates, Eq. (12) yields:

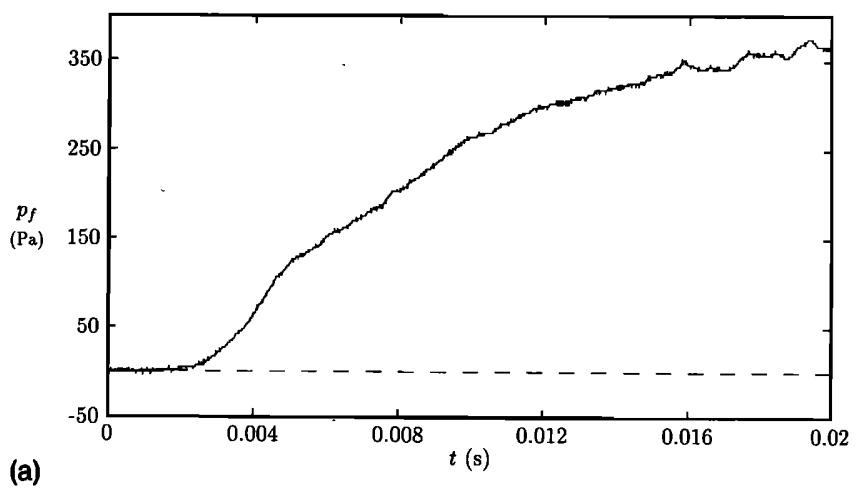

(a)

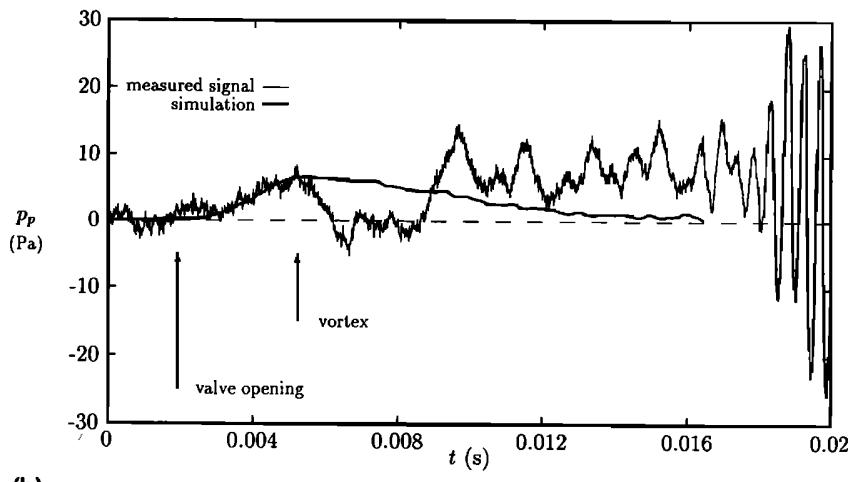

(b)

FIG. 11. Measured and simulated pressure signal for a slow valve opening: (a) measured pressure signal in the foot of the pipe; (b) measured pressure signal at the entrance of the pipe and corresponding simulated pressure signal.

$$
\rho_{0} \frac{l_{c}}{S_{e}} \frac{d Q_{j}}{d t} \approx p_{f}-p_{m}
$$

where $S_{e}=h_{e} H$ is the cross section of the channel at the flue exit. Similar relationships are obtained by applying the Bernoulli equation between points located at the exterior of the mouth, the entrance of the pipe and the flue exit:

$$
\begin{aligned}
& \rho_{0} \frac{\delta_{\text {out }}}{S_{m}} \frac{d Q_{\text {out }}}{d t} \approx p_{m}, \\
& \rho_{0} \frac{\delta_{\text {in }}}{S_{m}} \frac{d Q_{\text {in }}}{d t} \approx p_{m}-p_{p},
\end{aligned}
$$

where $\delta_{\text {out }}$ and $\delta_{\text {in }}$ are the portion of the end correction $\delta_{m}$ on the left and right of the origin and $p_{p}$ is the pressure signal at the entrance of the resonator. The end corrections $\delta_{\text {out }}$ and $\delta_{\text {in }}$ represent, respectively, the inertia at the mouth exit and the entrance of the pipe. As a first approximation it will be assumed, if we consider that the flue exit is located in the middle of the mouth constriction, that $\delta_{\text {in }}$ is equal to half of the end correction $\delta_{c}$ associated with the mouth constriction and that $\delta_{\text {out }}$ is equal to $\delta_{m}$ minus $\delta_{\text {in }}$ $\left(\delta_{m}=\delta_{\text {in }}+\delta_{\text {out }}\right)$. For times shorter than a round trip time of a sound wave traveling in the resonator (about $2 \mathrm{~ms}$ in our pipe) and neglecting friction in the pipe:

$$
p_{p} \approx \rho_{0} c_{0}\left(Q_{\mathrm{in}} / S_{p}\right) \text { : }
$$

By using Eqs. (15) $-(17)$ and the rise time $\tau$ of the driving pressure rise to estimate the time derivatives $(d Q /$ $d t \approx Q / \tau)$, the following relationship is obtained: 


$$
\frac{Q_{\text {out }}}{Q_{\text {in }}} \approx \frac{\delta_{\text {in }}}{\delta_{\text {out }}}+\frac{c_{0} \tau}{\delta_{\text {out }}} \frac{S_{m}}{S_{p}}
$$

Typical rise times $\tau$ in our experiments lie between 0.5 and $10 \mathrm{~ms}$ which implies that, for our experimental conditions, most of the flow $Q_{j}$ will be flowing out through the mouth. Hence, this very simple model qualitatively explains the strong deflection of the jet towards the exterior of the mouth. Furthermore, Eq. (18) shows that the fraction of the jet flow going outwards will increase, because of lower inertia, when decreasing the steepness of the driving pressure rise and inversely. This is in agreement with the observations we made from the flow visualizations and enables to relate the jet trajectory to the steepness of the pressure rise. Prediction of the jet deflection based on this ratio will be presented in Sec. VB. We now make the following approximations:

$$
\begin{aligned}
& Q_{\text {out }} \approx Q_{j}, \\
& p_{m} \approx p_{p},
\end{aligned}
$$

which are used in Eq. (15) to deduce the amplitude of the initial pulse:

$$
p_{p} \approx \frac{\rho_{0} \delta_{\text {out }}}{S_{m}} \frac{d Q_{j}}{d t}
$$

The pressure signal $p_{p}$ at the entrance of the pipe therefore depends on the time derivative of the jet flow $Q_{j}$. Velocity profiles such as the ones of Fig. 9 will result in a pressure pulse whose maximum will be reached at the moment of maximum jet velocity variations. It is now possible, by using Eqs. (14), (20), and (21), to express the pressure signal $p_{p}$ at the entrance of the pipe, for a given geometry of the mouth, as a function of the driving pressure $p_{f}$ only:

$$
p_{p} \approx p_{f} /\left(1+\frac{l_{c}}{\delta_{\text {out }}} \frac{S_{m}}{S_{e}}\right) \text {. }
$$

For our experimental pipe, Eq. (22) is equivalent to

$$
p_{p} \approx 0.06 p_{f} \text {. }
$$

Using the data of Figs. 10(a) and 11(a), the amplitude of the pressure pulses seen in Figs. 10(b) and Figs. 11(b) and predicted by Eq. (23) are 42 and $7 \mathrm{~Pa}$, respectively which is a fair approximation. The model just presented is only valid for times such that the movement of the jet is dominated by inertia and does not take into account the reflection of acoustic waves by the pipe. A more accurate model is presented in the following section.

\section{Pipe response including reflections}

The model presented in this section is based on the one-dimensional model shown in Fig. 7. The pressure $p_{m}$ at coordinate $x=-\delta_{\text {in }}$ is determined by the value of the lowfrequency approximation of the radiation impedance $Z_{\text {out }}$ at the mouth: ${ }^{24}$

$$
Z_{\text {out }}=\rho_{0} c_{0}\left[\frac{1}{4}\left(\frac{\omega}{c_{0}} r_{m}\right)^{2}+i \frac{\omega}{c_{0}} \delta_{\text {out }}\right],
$$

where $\omega$ is the angular frequency, $r_{m}$ the radius of a circle having the same cross section as that of the mouth $\left(\pi r_{m}^{2}=W H=S_{m}\right)$ and $\delta_{\text {out }}$ the end correction calculated in the previous section. Written in the time domain, by using inverse Fourier transform, Eq. (24) becomes

$$
p_{m}=\frac{\rho_{0} c_{0}}{S_{m}}\left(-\frac{1}{4} \frac{r_{m}^{2}}{c_{0}^{2}} \frac{d^{2} Q_{\mathrm{out}}}{d t^{2}}+\frac{\delta_{\mathrm{out}}}{c_{0}} \frac{d Q_{\mathrm{out}}}{d t}\right)
$$

The pressure wave $p_{p}$ inside the resonator is represented by two progressive waves traveling along the $x$ coordinate in opposite directions. In the frequency domain, it is equal to

$$
\hat{p}_{p}=\left(\hat{a} e^{-k x}+\hat{b} e^{k x}\right) e^{j \omega t},
$$

where $k$ is the complex propagation constant, $\hat{a}$ and $\hat{b}$ are complex amplitudes and are related by

$$
\hat{b}=R(\omega) \hat{a},
$$

where $R(\omega)$ is the reflection coefficient. Using the $x$-momentum equation and Eq. (26), the following expression for the acoustic flow $\hat{Q}_{p}$ inside the resonator is obtained:

$$
\hat{Q}_{p}=\frac{S_{p}}{Z_{c}(\omega)}\left(\hat{a} e^{-k x}-\hat{b} e^{k x}\right) e^{j \omega t},
$$

where $Z_{c}(\omega)$ is the characteristic impedance of the pipe. Using Eqs. (26)-(28) we find a relationship between the acoustic pressure $\hat{p}_{p}$ and the acoustic flow $\hat{Q}_{p}$ inside the pipe:

$$
\hat{p}_{p}=\frac{Z_{c}(\omega)}{S_{p}} \hat{Q}_{p}+R(\omega)\left(\frac{Z_{c}(\omega)}{S_{p}} \hat{Q}_{p}+\hat{p}_{p}\right) .
$$

This expression can now be written in the time domain by using the convolution theorem:

$$
\begin{aligned}
p_{p}(0, t)= & F^{-1}\left\{Z_{c}\right\} * \frac{Q_{\text {in }}}{S_{p}}+F^{-1}\left\{R(\omega) Z_{c}\right\} * \frac{Q_{\text {in }}}{S_{p}} \\
& +r(t) * p_{p}(0, t),
\end{aligned}
$$

where $F^{-1}$ denotes the inverse Fourier transform, $*$ the convolution integral and $r(t)$ is the reflection function at the entrance of the pipe defined as the inverse Fourier transform of the reflection coefficient. In our calculations, we used the following reflection function, proposed by Polack, ${ }^{22}$ which takes into account visco-thermic dissipation in the resonator but neglects radiation losses at the end of the resonator:

$$
r(t)= \begin{cases}\frac{-\alpha L}{r_{p} \sqrt{\pi}} \frac{e-\left[(\alpha L)^{2} / r_{p}^{2}(t-\tau)\right]}{(t-\tau)^{3 / 2}}, & \text { for } t>\tau \\ 0, & \text { for } t \leqslant \tau,\end{cases}
$$

with $L=L_{p}+\delta_{p}, \tau=2 L / c_{0}, r_{p}=H / \sqrt{\pi}$ and:

$$
\alpha=\frac{\left[\sqrt{l_{v}}+(\gamma-1) \sqrt{l_{t}}\right]}{\sqrt{c_{0}}},
$$

where $l_{v}$ and $l_{t}$ are the viscous and thermal characteristic lengths (in air at $20^{\circ} \mathrm{C}, \quad l_{v}=4.0 \times 10^{-8} \mathrm{~m}$ and 
$\left.l_{t}=5.6 \times 10^{-8} \mathrm{~m}\right)$ and $\gamma$ is the Poisson constant, ratio of specific heats at constant pressure and volume, respectively $(\gamma=1.4)$. One has to be careful with the use of this reflection function for calculation of the flow in the pipe since this function will allow an arbitrary de component of $Q_{\text {in }}$. This behavior does not enable one to determine correctly the flow repartition at the flue exit. In a more elaborate model that includes the oscillations of the jet, it would also cause problems in determining the jet displacement. This issue is discussed extensively in the study of Valeriu. ${ }^{25}$

For the sake of simplicity, we assumed in our calculations that friction has a negligible effect on the value of the characteristic impedance $Z_{c}$ and therefore that it is equal to $\rho_{0} c_{0}$. We do not have, at the present time, a clear idea of the implications of this approximation on the solution of the problem. Equation (30) then simplifies to

$$
p_{p}(0, t)=\frac{\rho_{0} c_{0}}{S_{p}} Q_{\text {in }}+r(t) *\left(\frac{\rho_{0} c_{0}}{S_{p}} Q_{\text {in }}+p_{p}(0, t)\right),
$$

which is similar to the expression proposed by McIntyre et $a l^{3}$

To complete the model the pressure at the origin is related to the pressure at the entrance of the pipe by taking into account the effects of inertia at the entrance of the resonator:

$$
p_{m}-p_{p}(0, t)=\frac{\rho \delta_{\text {in }}}{S_{m}} \frac{d Q_{p}(0, t)}{d t} .
$$

The flow $Q_{j}$ is computed from Bernoulli's equation [Eq. (12)] and the value of the pressure $p_{m}$ in the mouth and $p_{f}$ in the foot of the pipe at the previous time step. The other parameters are found by solving simultaneously Eqs. (13), (25), (33), and (34).

Simulations of the pressure signals at the entrance of the pipe obtained with this model are compared to the experimental data in Figs. 10(b) and 11(b). In both cases, the amplitude of the initial pulse is close to that of the calculated pressure signal. Furthermore, the peak pressure is reached at the moment of maximum jet velocity variation as we should expect (see Fig. 9). The pressure then gradually drops towards zero as the jet velocity stabilizes. The reflected signal from the pipe is very clearly seen on the simulated pressure signal corresponding to the fast opening. In the case of the slow opening of the valve it is not apparent since the jet velocity variations still generate significant acoustic waves, which dominate the pressure signal, when the initial pulse comes back after a round trip in the resonator.

The simulated signals are in good agreement with the experimental data during the first milliseconds. Later on, in both cases, the pressure drops much more rapidly in the experimental data. Flow visualizations equivalent to the ones presented in Figs. 4 and 5 and corresponding to the pressure signals of Figs. 10 and 11 show that this moment coincides exactly with the initial jet-labium interaction. At this instant a vortex is shed at the labium which induces, as can be deduced from the direction of rotation of the vortex, a negative pressure pulse. This is followed by subsequent bursts at regular time intervals each time that the jet hits the labium. Consistently with the observations we made from the flow visualizations, the jet-labium interaction is delayed in the case of the manual opening of the valve. Our model, which ignores the formation of vortices at the labium is only accurate before the jet touches the labium. The simulations that we present however yield a quantitative information on the importance of vortex shedding during the initial jet-labium interaction.

\section{E. Jet velocity fluctuations during steady-state operation}

In models that describe the behavior of flue organ pipes, the jet velocity is usually considered to be constant during steady-state operation. The jet velocity fluctuations are, however, important and they provide acoustic work. The jet velocity is determined by the difference between the pressure $p_{m}$ in the mouth of the pipe exit and the pressure $p_{f}$ in the foot. During steady-state operation, the amplitude of the pressure measured under the labium is approximately equal to the mean pressure in the foot [see Fig. 2(a)-(b), and Fig. 3(a)-(b)]. The amplitude of the pressure signal $p_{m}$ at the flue exit is estimated to be equal to $2 / 3$ of that of the driving pressure $p_{f}$. This assumption is based on Coltman's ${ }^{26}$ acoustic pressure measurements in the mouth of a flue organ pipe similar to our pipe and will be used throughout this paper. These pressure variations can therefore be expected to induce significant fluctuations of the jet velocity.

An equation describing the jet velocity fluctuations can be found by using the Bernoulli equation. If the different variables are represented as the sum of a mean value $\langle x\rangle$ and a time dependant term $x^{\prime}$, Eq. (12) becomes after linearization

$\rho_{0} l_{c} \frac{d v_{j}^{\prime}}{d t}+\frac{1}{2} \rho_{0}\left\langle v_{j}\right\rangle^{2}+\rho_{0}\left\langle v_{j}\right\rangle v_{j}^{\prime}+p_{m}^{\prime}=\left\langle p_{f}\right\rangle+p_{f}^{\prime}$.

By separating the steady term and the first-order terms of the fluctuations in Eq. (35), the following relationships are obtained

$$
\begin{aligned}
& \left\langle p_{f}\right\rangle=\frac{1}{2} \rho_{0}\left\langle v_{j}\right\rangle^{2}, \\
& \rho_{0} l_{c} \frac{d v_{j}^{\prime}}{d t}+\rho_{0}\left\langle v_{j}\right\rangle v_{j}^{\prime}+p_{m}^{\prime}=p_{f}^{\prime} .
\end{aligned}
$$

If it is supposed that the foot of the pipe is supplied by a constant flow source $S_{e} \rho_{0}\left\langle v_{j}\right\rangle$, the mass conservation law applied between the entrance of the foot and the flue exit gives, after linearization:

$$
S_{e}\left(\rho_{0} v_{j}^{\prime}+\rho_{m}^{\prime}\left\langle v_{j}\right\rangle\right)=-V_{f} \frac{d \rho_{f}^{\prime}}{d t},
$$

where $V_{f}$ is the volume of the foot and $\rho_{m}^{\prime}$ and $\rho_{f}^{\prime}$ the density fluctuations in the mouth and the foot of the pipe. Please note that the constant flow source has been chosen as the most simple model allowing foot pressure fluctuations $p_{f}^{\prime}$. The main results of the analysis will be quite independant of this assumption. Eliminating the densities in Eq. (38) with the constitutive equation $p^{\prime}=c_{0}^{2} \rho^{\prime}$ and 
combining this new equation with the time derivative of Eq. (37), the following relationship is obtained

$$
\frac{d^{2} v_{j}^{\prime}}{d t^{2}}+\Gamma \frac{d v_{j}^{\prime}}{d t}+\omega_{0}^{2} v_{j}^{\prime}=-\frac{1}{\rho_{0}^{\prime} c} \frac{d p_{m}^{\prime}}{d t}-\frac{\omega_{0}^{2}\left\langle v_{j}\right\rangle}{\rho_{0} c_{0}^{2}} p_{m}^{\prime},
$$

where

$$
\omega_{0}^{2}=\frac{c_{0}^{2} S_{e}}{V_{f} l_{c}} \text { and } \Gamma=\frac{\left\langle v_{j}\right\rangle}{l_{c}} .
$$

The foot therefore acts like a damped Helmholtz resonator with natural angular frequency $\omega_{0}$ and a quality factor inversely proportional to the mean jet velocity. It is interesting to note that this simple model explains qualitatively the mouth response observed by Coltman. ${ }^{27}$

The damping factor, which is induced by the mean flow, is somehow unexpected since a loss mechanism was not assumed explicitly. The damping was in fact introduced when the pressure at the flue exit was assumed to be the same as the pressure $p_{m}$ at the mouth. Indeed, this is due to the formation of a jet corresponding to a Kutta condition $^{23}$ at the edges of the flue exit. This condition states that the velocity at an edge should remain finite which implies tangential flow separation and the shedding of vorticity in the main flow at the boundary between the moving fluid and stagnant fluid outside the jet. In fact the Kutta condition is a trick which allows to include within the framework of a frictionless theory a consequence of the local action of friction at a sharp edge: flow separation which is responsible for the formation of the free jet. The jet velocity fluctuations result into a modulation of the vorticity shed at the edges which implies the loss of kinetic energy for the acoustic field as shown by Cummings and Eversman. ${ }^{28}$

The amplitude of the jet velocity fluctuations can be calculated from Eqs. (36) and (37). Assuming harmonic motion, $x^{\prime}=\hat{x} e^{i \omega t}$, where $\hat{x}$ is a complex amplitude, the following relationship is obtained:

$$
\frac{\hat{v}_{j}}{\left\langle v_{j}\right\rangle} \approx \frac{\left(\hat{p}_{f}-\hat{p}_{m}\right)(1-i \mathrm{Sr})}{2\left\langle p_{f}\right\rangle\left(1+\mathrm{Sr}^{2}\right)},
$$

with

$$
\mathrm{Sr}=\frac{\omega l_{c}}{\left\langle v_{j}\right\rangle},
$$

where $\mathrm{Sr}$ is the Strouhal number. For typical experimental conditions $\left(\left\langle p_{f}\right\rangle=180 \mathrm{~Pa}\right)$, the Strouhal number for our organ pipe is of the order of 5 . This yields, assuming that the pressure fluctuations $p_{f}^{\prime}$ in the foot can be neglected which implies that the playing frequency is far from the natural angular frequency $\omega_{0}$ of the foot of the pipe, that the component of the jet velocity fluctuations in opposition of phase with the acoustic movement at the mouth represents about $20 \%$ of the amplitude of the total fluctuations. The flue channel length is seen to have a considerable influence on the amplitude of the jet velocity fluctuations since the Strouhal number is proportional to this parameter. When the channel length is decreased, the component of the jet velocity fluctuations out of phase with the acous- tic movement increases because of a smaller inertia in the channel. The volume of the foot is another parameter that can be used to control the velocity fluctuations since it determines its natural frequency and, hence, the amplitude of the pressure variations in the foot of the pipe.

The average power $\left\langle P_{v_{i}^{\prime}}\right\rangle$ dissipated by the jet velocity fluctuations can be calculated from Eq. (40), experimental measurements of the pressure difference between the foot and the mouth of the pipe and the following expression:

$$
\left\langle\boldsymbol{P}_{v_{j}^{\prime}}\right\rangle_{T}=\frac{S_{e}}{2} \operatorname{Re}\left(\hat{p}_{m}^{*} \hat{v}_{j}\right)
$$

A simple explicit formulation of the average power $\left\langle\boldsymbol{P}_{v_{j}^{\prime}}\right\rangle$ is found if the pressure variations in the foot can be neglected compared to the pressure variations at the mouth and if the fundamental is assumed to dominate. Furthermore, for our experimental pipe, the amplitude of the acoustic signal at the flue exit is estimated to be of the order of $2 / 3$ of the mean driving pressure $\left\langle p_{f}\right\rangle$. Using these approximations and Eqs. (36), (40), and (42), the following expression is found

$$
\left\langle\boldsymbol{P}_{v_{j}^{\prime}}\right\rangle_{T} \approx \frac{-\sqrt{2} S_{e}\left\langle p_{f}\right\rangle^{3 / 2}}{9 \sqrt{\rho_{0}}\left(1+\mathrm{Sr}^{2}\right)} .
$$

The power dissipated by the jet velocity fluctuations increases nonlinearily with the driving pressure $\left\langle p_{f}\right\rangle$ and decreases nonlinearly with the frequency. Calculations made from Eq. (43) for our experimental pipe and for a mean driving pressure $\left\langle p_{f}\right\rangle$ of $180 \mathrm{~Pa}$ give losses of the order of $3 \times 10^{-4} \mathrm{~W}$.

The importance of the losses associated with the fluctuating jet velocity can be appreciated by comparing them to the loss mechanisms usually considered, that is to say the radiative and visco-thermic losses. The power radiated by the fundamental calculated by $\mathrm{Fabre}^{6}$ for the same experimental conditions as precedently, is equal to $8 \times 10^{-5}$ W. For the fundamental the visco-thermic losses are approximately three times as important as the radiative losses $^{6}$ which give losses of approximately $2.5 \times 10^{-4} \mathrm{~W}$. These calculations show that the losses associated with the jet velocity fluctuations can be expected to be of the same order of magnitude as the radiative and visco-thermic losses. They, therefore, represent a non-negligible damping mechanism for the acoustic oscillations in the resonator. More accurate calculations based on the experimentally measured foot and pipe pressures do not radically change the conclusions obtained with the simple model proposed as long as the foot pressure fluctuations $p_{f}^{\prime}$ are smaller than the pressure fluctuation $p_{m}^{\prime}$ at the mouth.

\section{JET FORMATION TIME AND INITIAL DEFLECTION}

\section{A. Jet formation time}

For a time-domain simulation of the transient of flue organ pipes that would take into account the effects of vortex shedding at the labium, it is important to be able to determine the moment at which the forming jet reaches the labium. This can be done by different methods. Experimen- 


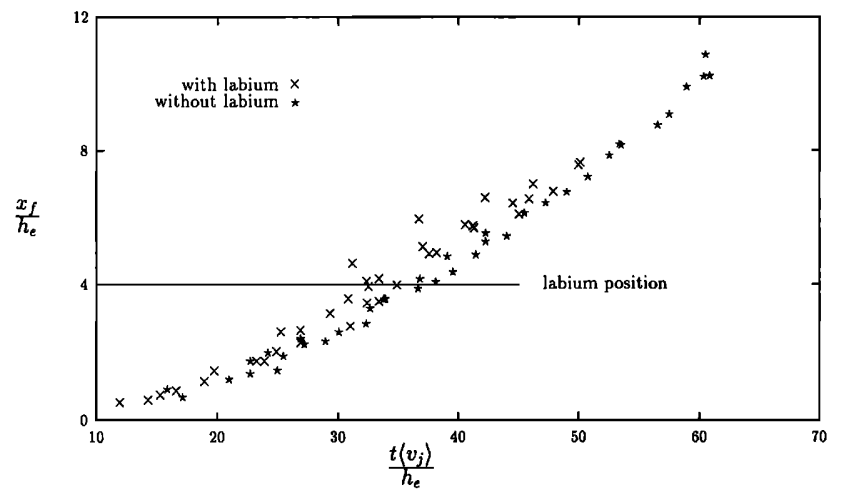

FIG. 12. Experimental measurements of the horizontal position of the jet front with and without labium for a mechanical opening of the valve.

tally, it appears that the labium has no influence on the front position before it reaches the labium. Measurements of the front horizontal position $x_{f}$ with and without the labium are presented in Fig. 12 and the two data series clearly overlap. Its presence will, therefore, be neglected for the estimation of the horizontal position.

An upper limit is calculated by assuming flow separation at the flue exit and a uniform jet width. The front position $\left(x_{f}\right)_{\max }$ is then given by a simple time integration of the jet velocity at the flue exit:

$$
\left(x_{f}\right)_{\max }=\int_{0}^{t} v_{j} d \tau,
$$

where the velocity $v_{j}$ is calculated for given pressure signals $p_{f}$ and $p_{m}$ by using the Bernoulli equation (see Sec. IV B). A lower limit is found by assuming that the flue exit acts as a point source of a potential flow $Q_{j}(t)$ flowing in a semi-infinite space. The jet front position $\left(x_{f}\right)_{\min }$ is then given (in a two-dimensional representation) by the following relationship:

$$
\frac{d\left(x_{f}\right)_{\min }}{d t}=\frac{v_{j} h_{e}}{\pi\left(x_{f}\right)_{\min }},
$$

which is equivalent to

$$
\left(x_{f}\right)_{\min }=\left(\frac{2 h_{e}}{\pi} \int_{0}^{t} v_{j} d \tau\right)^{1 / 2}=\left(\frac{2 h_{e}}{\pi}\left(x_{f}\right)_{\max }\right)^{1 / 2} .
$$

The front position $\left(x_{f}\right)_{\max }$ or $\left(x_{f}\right)_{\min }$ can therefore be calculated if the jet velocity is known. An approximation of the velocity $v_{j}$ is found for small times, such that the effects of inertia dominate, by keeping only the first term in the right-hand side of Eq. (12):

$$
v_{j}=\int_{0}^{t}\left(\frac{p_{f}-p_{m}}{\rho_{0} l_{c}}\right) d \tau
$$

Assuming a constant foot pressure $p_{f}$ and neglecting the pressure $p_{m}$ in the mouth, we obtain

$$
\left(x_{f}\right)_{\max }=\frac{p_{f}}{2 \rho_{0} l_{c}} t^{2}
$$

and

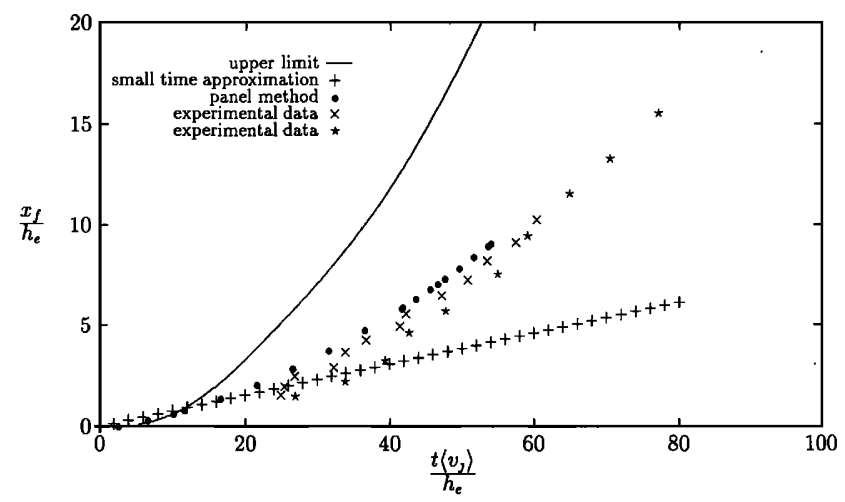

FIG. 13. Horizontal displacement of the jet predicted by different models for a mechanical opening of the valve and comparison with experimental measurements.

$$
\left(x_{f}\right)_{\min }=t \sqrt{\frac{p_{f} h_{e}}{\pi \rho_{0} l_{c}}}
$$

A better estimation of the front position is obtained by using a more elaborate two-dimensional model, called the second-order panel method, ${ }^{29}$ which takes into account the formation of vortices at both edges of the flue exit. The boundaries of the jet are represented by lines along which the vorticity is concentrated. The velocity induced by the vorticity distribution is calculated by using the Biot Savart induction law. The front displacement can then be determined by integration of the velocity.

The vortex front position calculated with those three methods as well as experimental measurements are presented in Fig. 13 for a mechanical opening of the valve. The initial displacement of the front is well predicted by the three methods. As the front progresses, only the panel method remains accurate, and its position lies between the one predicted by the two simple models described above.

\section{B. Initial Jet deflection}

As was seen from the flow visualizations presented in Sec. III, the forming jet initially bends towards the exterior of the pipe. This deflection is stronger for a slow pressure rise in the foot than for a steep one. As was discussed previously, this jet behavior seems to play an important role in the triggering of the transient since it determines whether the jet will hit or miss the labium. In order to determine whether there will initially be a contact between the jet and the labium, the vertical position of the jet when it passes the labium must therefore be calculated.

The jet deflection can be viewed as being a consequence of the asymmetric flow distribution between the exterior and the interior of the pipe. In Sec. IV C this flow distribution was explained in terms of the difference between the low inertia of the air just outside the mouth and the compressibility of the air at the entrance of the pipe. This asymmetry between the flows $Q_{\text {in }}$ and $Q_{\text {out }}$ forces the jet to move towards the exterior of the pipe. The vertical distance traveled by the jet, when it reaches the labium, is therefore determined by the geometry of the mouth and the acceleration of the jet. 


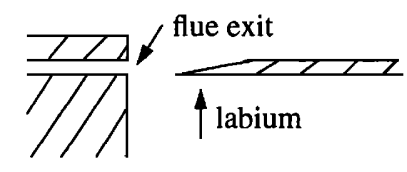

(a)

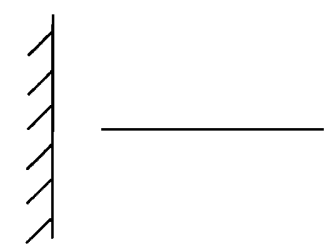

(b)

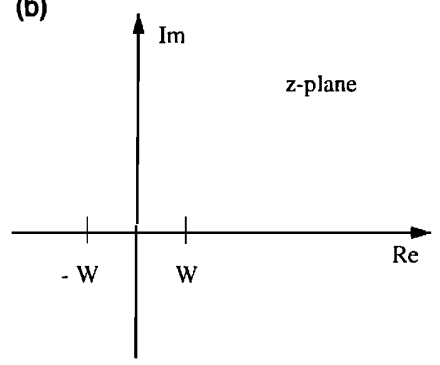

(c)

FIG. 14. Idealized geometry of mouth of an organ pipe used for conformal mapping: (a) actual geometry; (b) simplified geometry; and (c) z-plane configuration for calculations.

It is possible, using the potential flow theory, to simulate this jet deflection if we consider the mouth as a diaphragm opening between a vertical wall and a half-infinite horizontal plane representing the labium. The model proposed here is very crude but nevertheless enables to reproduce qualitatively the initial behavior of the jet. By using the Schwarz-Christoffel conformal mapping and the method of images, the following mapping of the idealized geometry of Fig. 14 in the $z$ plane into the upper-half of the $w$ plane is found:

$$
\begin{aligned}
& z=\frac{W}{2}\left(w+\frac{1}{w}\right), \\
& w=\frac{z}{W} \pm \sqrt{\left(\frac{z}{W}\right)^{2}-1,}
\end{aligned}
$$

where $W$ is the distance between the origin and the labium. The jet flow at the flue exit is represented by a point source $Q_{j}$ located at coordinate $\left(0, y_{0}\right)$ and the portion of the jet flow going into the pipe by a sink $Q_{\text {in }}$ located at infinity. This source and sink determine a velocity field from which the trajectory of a particle released at the flue exit can be calculated.

An estimation of the jet deflection associated with the driving pressure histories shown in Sec. II can be calculated by using the results of the acoustic simulations analogous to the one presented in Sec. IV D. At every time step, the value of the point source and sink are replaced by the calculated value of the jet flow $Q_{j}$ and the portion $Q_{\text {in }}$ that goes into the pipe, hence, introducing the time dependency of the source and sink. At regular time intervals,

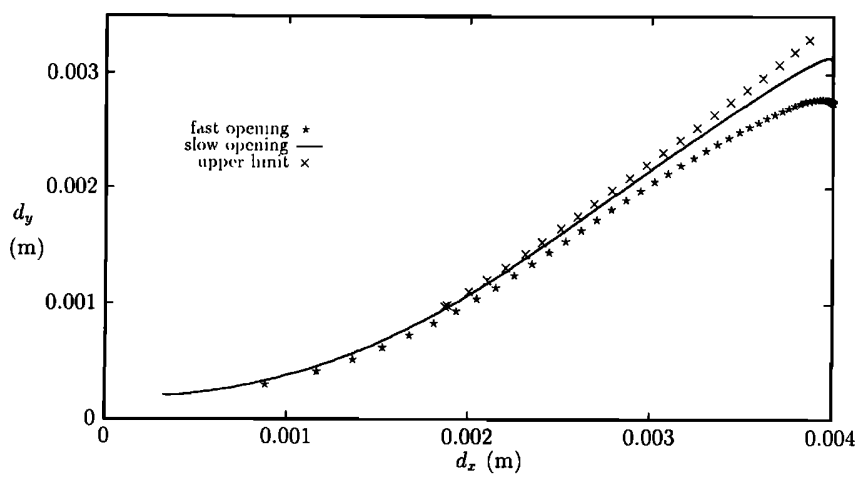

FIG. 15. Jet deflection predicted by potential flow theory for the fast and slow valve opening of the valve. The upper limit is calculated by assuming $Q_{\text {in }}=0$ in the case of the slow valve opening.

new particles are released at the flue exit. The deflection of the jet is found by plotting the position $\left(d_{x}, d_{y}\right)$ of every released particle at the moment when the horizontal distance traveled by the first one is equal to the distance between the flue exit and the labium.

Results obtained by using this method are shown in Fig. 15. In this example, the returning flow from the pipe was neglected in the computation of the flow $Q_{\text {in }}$. Indeed, as was pointed out in Sec. IV D, the reflection function we use allows a dc component in the flow to propagate in the pipe which does not enable to evaluate correctly the flow repartition at the flue exit. As expected, the vertical distance traveled by the jet, when it reaches the labium, is greater in the case of the slow driving pressure rise than with the steep one. However, in both cases, this distance is too high in comparison with the flow visualizations, especially in the case of steep pressure rise. The height difference between the two curves at abscissa $4 \mathrm{~mm}$ is about 0.4 $\mathrm{mm}$. This value represents about half of the value observed in the flow visualizations presented in Sec. III, where the deflection difference between the two series of pictures is of the order of $1 \mathrm{~mm}$. The fact that the predicted jet deflection $y$ is too large is not unexpected, because the model corresponds to the calculation of the horizontal position $x_{\min }$. Hence, the arrival time of the jet at the labium is an upper bound. The major concern is that the difference between the behaviors for fast and slow pressure rises is too small. In order to increase the deflection difference, we had to assume that the jet flow is directed entirely towards the exterior of the pipe through the mouth $\left(Q_{\text {in }}=0\right)$. The upper limit in Fig. 15 was calculated with this assumption in the case of a slow valve opening. More elaborated simulations, taking into account complex aspects of the flow such as vortex formation and friction at the walls and using the same values of the flow distribution at the flue exit as in the example of Fig. 15, predicted jet deflections close to the experimental measurements but still a too small height difference between the steep and smooth pressure rise. This constitutes a limitation of the simple acoustic model of Fig. 7. Indeed, even though it is able to predict the amplitude of the pressure pulse due to the initial volume injection, it 
only enables to describe qualitatively the initial movement of the jet.

\section{CONCLUSION}

In this paper we have related different events of the jet formation to the pressure signal measured at the entrance of the pipe during the first phase of the initial transient. Using a simple acoustic model, it was shown that the volume injection into the mouth of the pipe which accompanies the formation of the jet provides the initial acoustic wave at the entrance of the resonator. The flow visualizations presented in this paper show that the forming jet bends towards the exterior of the pipe. This behavior is related to an asymmetric flow distribution between the exterior and interior of the pipe caused by the difference between the low inertia of the air at the mouth and the compressibility of the air at the entrance of the resonator. The vertical height reached by the jet when it passes at the level of the labium was related to the steepness of the pressure rise. This is an important parameter to consider since it determines whether the jet hits the labium. Indeed, when it does, a vortex is shed at the labium which drastically affects the shape of pressure signal during the attack transient. It is difficult, once the jet has touched the labium, to relate precisely the events of the flow visualization to the pressure signal. Indeed, the returning signal from the pipe has to be taken into account and the newly formed jet is unstable.

These features of the initial jet behavior could explain why organ builders use ears around the mouth of the pipe. The ears, by increasing the inertia around the mouth, have two effects. First, they increase the amplitude of the initial pressure pulse and second they force the jet to follow a straighter trajectory and to hit the labium. They therefore contribute to produce a sharper transient with richer harmonic content. Hence by using ears, the organ buider can obtain a sharp attack transient with a relatively low labium position. This position of the labium is crucial for the amount of even harmonics generated in the steady-state sound spectra. ${ }^{2,6}$

During steady-state operation, the pressure variations at the flue exit were found to induce non-negligible jet velocity fluctuations. This phenomenon results into a damping mechanism that increases nonlinearly with the driving pressure. As the phase difference between the jet velocity fluctuations and the acoustic pressure at the mouth is determined by the inertia of the air mass in the flue channel, the length of this channel determines the importance of these losses. This could be a reason why recorders generally have long flue channels.

\section{ACKNOWLEDGMENTS}

The authors would like to thank Dr. René Caussé, Dr. Michèle Castellengo, Dr. Joël Gilbert, and Leonie Schoenmakers for useful discussions and their support throughout this work. This work is part of the thesis work of Marc-Pierre Verge which is carried out under the supervision of Dr. J. Kergomard of the Université du Maine (Le Mans, France).
${ }^{1} \mathrm{~L}$. Cremer and $\mathrm{H}$. Ising, "Die selbsterregten schwingungen von orgelpfeifen," Acustica 19, 143-153 (1967).

${ }^{2}$ N. H. Fletcher and T. D. Rossing, The Physics of Musical Instruments (Springer-Verlag, New York, 1991).

${ }^{3}$ M. E. McIntyre, R. T. Schumacher, and J. Woodhouse, "On the oscillations of musical instruments," J. Acoust. Soc. Am. 74, 1325-1345 (1983).

${ }^{4} \mathrm{~S}$. A. Elder, "On the mechanism of sound production in organ pipes," J. Acoust. Soc. Am. 54, 1554-1564 (1973).

${ }^{5}$ N. H. Fletcher, "Jet-drive mechanism in organ pipes," J. Acoust. Soc. Am. 60, 481-483 (1976).

${ }^{6}$ B. Fabre, Ph.D. thesis, Université du Maine, Le Mans, France, 1992.

${ }^{7} \mathrm{~J}$. W. Coltman, "Sounding mechanism of the flute and organ pipe," J. Acoust. Soc. Am. 44, 983-992 (1968).

${ }^{8} \mathrm{M}$. S. Howe, "Contribution to the theory of aerodynamics sound, with application to excess jet noise and the theory of the flute," J. Fluid Mech. 71, 625-673 (1975).

${ }^{9}$ W. Merzkirch, Flow Visualization (Academic, New York, 1974).

${ }^{10} \mathrm{~A}$. W. Nolle and T. L. Finch, "Starting transients of flue organ pipes in relation to pressure rise time," J. Acoust. Soc. Am. 91, 2190-2202 (1992).

"N. H. Fletcher, "Transients in the speech of organ flue pipes-a theoritical study," Acustica 34, 224-233 (1976).

${ }^{12} \mathrm{~J}$. Angster and A. Miklos, "New developments on the understanding of the buildup of organ sounds," in Proceedings of the 13th International Congress on Acoustics, Belgrade, 1989 (unpublished).

${ }^{13} \mathrm{M}$. P. Verge and R. Caussé, "Linear analysis of the initial transient of a recorder," in Proceedings of the Stockholm Musical Acoustics Conference, Royal Swedish Academy of Music, 1993 (unpublished).

${ }^{14}$ A. Powell, "Some aspects of aeroacoustics from Rayleigh until today," J. Vib. Acoust. 112, 145-159 (1990).

${ }^{15}$ Sato H, "The stability and transition of a two-dimensional jet," J. Fluid Mech. 7, 53-80 (1960).

${ }^{16}$ R. Kaykayoglu and D. Rockwell, "Unstable jet-edge interaction. Part 1. instanteneous pressure field at a single frequency," J. Fluid Mech. 169, 125-149 (1986).

${ }^{17}$ A. Hirschberg, A. van Steenbergen, A. P. J. Wijnands, and J. J. de Vries, "Jet drive and edgetone in flue organ pipes," in Colloque Modèles Physiques (INPG, Grenoble, France, 1990), ACROE.

${ }^{18}$ M. Castellengo, Ph.D. thesis, Université de Paris VI, Paris, France, 1976.

${ }^{19} \mathrm{~A}$. van Steenbergen, "Endcorrection and resonance frequencies of the flue organ-pipe," Technical Rep. R-1046-S, Eindhoven University of Technology, The Netherlands, January 1990.

${ }^{20}$ P. M. Morse and K. U. Ingard, Theoretical Acoustics (McGraw-Hill, New York, 1968).

${ }^{21}$ M. C. A. M. Peters, A. Hirschberg, A. J. Reijnen, and A. P. J. Wijnands, "Damping and reflection coefficient measurements for an open pipe at low mach and low helmholtz numbers," J. Fluid Mech. 256, 499-535 (1993).

${ }^{22}$ J. D. Polack, X. Menial, J. Kergomard, C. Cosnard, and M. Bruneau, "Reflection function of a plane wave in a cylindrical tube," Revue Phys. Appl. 22, 331-337 (1987).

${ }^{23}$ A. R. Paterson, A First Course in Fluid Dynamics (Cambridge U.P., Cambridge, 1983).

${ }^{24} \mathrm{~J}$. W. Strutt and B. Rayleigh, The Theory of Sound (Dover Reprint, New York, 1954).

${ }^{25}$ I. Valeriu, Master's thesis, University of Washington, Seattle, WA, 1992.

${ }^{26} \mathrm{~J}$. W. Coltman, "Jet drive mechanism in edge tones and organ pipes," J. Acoust. Soc. Am. 60, 725-733 (1976).

${ }^{27} \mathrm{~J}$. W. Coltman, "Mouth resonance effects in the flute," J. Acoust. Soc. Am. 54, 417-420 (1973).

${ }^{28} \mathrm{~A}$. Cummings and W. Eversman, "High amplitude acoustic transmission through duct terminations: Theory," J. Sound Vib. 91, 503-518 (1983).

${ }^{29}$ H. W. M. Hoeijmakers, Ph.D. thesis, Technische Universiteit Delft, Delft, The Netherlands, 1989. 